\title{
The theory of electron diffraction by the void lattice
}

\author{
A M Stoneham \\ Theoretical Physics Division, AERE Harwell, Berkshire, UK
}

Received 22 January 1974, in final form 27 February 1974

\begin{abstract}
The theory is given for the electron diffraction by the void lattice in molybdenum. recently observed by Sass and Eyre. The results are analysed to see if this method can give useful new information about the voids and their ordering.

The predictions of the positions and intensities of the extra peaks agree with observation. However, the quantitative theory shows that void shapes cannot readily be found from the diffraction data. Nor is it easy to get accurate information about void sizes or the nature of the disorder in the void lattice, mainly because of the problems of measuring intensity profiles. The effects of the various forms of lattice disorder are discussed qualitatively.
\end{abstract}

\section{Introduction}

Recent work has established that periodic arrays of voids are formed in metals after suitable irradiation (Evans 1971, Eyre and Evans 1971, Wiffen 1972, Kulcinski et al 1972). The regularity of these remarkable three dimensional arrays was exploited recently by Sass and Eyre (1973), who observed electron diffraction from void and bubble lattices in irradiated molybdenum.

The theory of electron diffraction by the void lattice is discussed in the present paper. In principle the observed diffraction patterns contain useful information about the voids and about their ordering. The main aim of the present paper is to examine the possibility of obtaining this information in practice. One might hope to obtain estimates of the sizes of voids and the nature of their faceting for example. Or one might want to judge the degree and nature of the imperfection of the void lattice. Sass and Eyre used their data to obtain accurate estimates of the spacings of the voids in the lattice; here we concentrate on the other aspects.

\section{General theory}

The diffraction will be discussed using kinematic theory. Naturally one has reservations about using a theory assuming only weak diffraction and absorption for an electron beam in a metal. But the approximations are not violated too strongly for the thin films used, and, in any case, the main qualitative features should be given adequately by the simple theory. In particular, negative conclusions will surely hold in both theories: if kinematic theory indicates some parameter cannot easily be obtained from observed patterns, the same should be true for dynamical theory. 
In the kinematic theory, diffracted intensities are proportional to the square modulus of a matrix element $M(K)$ where $K$ is the scattering vector. If the electron is scattered by some array (however imperfect) of $N_{0}$ identical scatterers, then $M$ can be factorized:

$$
M(K)=V(K) S(K)
$$

(eg Ziman 1964, §2.7). One factor, $V(\boldsymbol{K})$, depends only on the nature of the scatterers. The other, the structure factor $S$, depends only on the spatial arrangement of the scatterers:

$$
S(\boldsymbol{K})=\frac{1}{N_{0}} \sum_{l} \exp \left(-\mathrm{i} \boldsymbol{K} \cdot \boldsymbol{R}_{l}\right) .
$$

Two consequences of the factorization are important here. First, since only one species is present in the systems of interest, one may usually concentrate on the structure factor only. Secondly, the structure factor of a system with vacancies can be written as the difference of two terms. One describes the lattice with no vacancies, and the other is determined by the distribution of the $n$ vacancies. This leads to a great simplification, since it is then straightforward to separate the contributions of the lattice defects to diffraction:

$$
\begin{aligned}
S(\boldsymbol{K}) & =\frac{1}{N_{0}} \sum_{\substack{\text { all sites } \\
\text { (f ull or } \\
\text { empty) }}} \exp \left(-\mathrm{i} \boldsymbol{K} \cdot \boldsymbol{R}_{l}\right)-\sum_{\substack{\text { empty } \\
\text { sites }}} \exp \left(-\mathrm{i} \boldsymbol{K} \cdot \boldsymbol{R}_{l}\right) \\
& =\frac{N}{N_{0}} S_{0}(\boldsymbol{K})-\frac{n}{N_{0}} \sigma(\boldsymbol{K})
\end{aligned}
$$

where $N \equiv N_{0}+n$ is the total number of sites. Here the structure factor of the perfect lattice is

$$
S_{0}(\boldsymbol{K})=\frac{1}{N} \sum_{\substack{\text { all sites } \\ l}} \exp \left(-\boldsymbol{K} \cdot \boldsymbol{R}_{l}\right)
$$

and that of the vacancy array is

$$
\sigma(\boldsymbol{K})=\frac{1}{n} \sum_{\substack{\text { cumply } \\ \text { sites } m}} \exp \left(-\mathrm{i} \boldsymbol{K} \cdot \boldsymbol{R}_{m}\right)
$$

There are two implicit assumptions. One is that form factors $V(\boldsymbol{K})$ of the atoms are unaltered by the presence of nearby vacancies. Whilst this is hard to assess, it is unlikely to be so important as to affect the present work greatly, and it is straightforward in principle (if not in practice) to verify. The other assumption is that we ignore interstitials, dislocations, impurity atoms and other defects. The reason is that there is no evidence for ordering and its consequences associated with these other imperfections. Their main effect is to broaden the observed peaks and to set bounds on the resolution of the experiments.

We now calculate structure factors $\sigma(K)$ in a number of cases of interest. It is useful to recall that the structure factor of a perfect infinite crystal is

$$
S_{0}(\boldsymbol{K})=\delta_{\boldsymbol{K}, \boldsymbol{\theta}_{H}}
$$

where $g_{\mathrm{H}}$ is a reciprocal lattice vector for the host crystal. 


\section{Structure factors for perfect void arrays}

For simplicity, an array of isolated vacancies is discussed first. None of the experimental results fits this picture, but the vacancy array is a useful reference system for the cases discussed later. The array is assumed to have the same structure as the host (eg bcc or fcc), but the spacing of the vacancy lattice is a factor $L$ larger than that of the host. Thus $N / n$ is $L^{3}$, and the reciprocal lattice vectors for the vacancy lattice are $g_{\mathrm{D}} \equiv g_{\mathrm{H}} / L$. The defect structure factor $\sigma(\boldsymbol{K})$ becomes:

$$
\sigma_{0}(\boldsymbol{K})=\delta_{\boldsymbol{K}, g_{\mathrm{D}}}
$$

When $\boldsymbol{K}$ coincides with a host vector $\boldsymbol{g}_{\mathrm{H}}$ (and hence also with a $\boldsymbol{g}_{\mathrm{D}}$ ) the total structure factor is

$$
S(\boldsymbol{K})=\frac{N}{N_{0}}-\frac{n}{N_{0}}=1 .
$$

Thus the vacancy array does not affect the intensity of the main peaks. The additional peaks occur when $\boldsymbol{K}$ coincides with a defect lattice vector $g_{\mathrm{D}}$ only, when

$$
S(\boldsymbol{K})=\frac{n}{N_{0}} .
$$

This is independent of which $g_{\mathrm{D}}$ is involved. The diffraction pattern expected consists of the usual and dominant host peaks, regularly interspersed with weaker peaks which are all of the same intensity.

\subsection{Infinite array of voids}

We now show that $\sigma(\boldsymbol{K})$ for an array of voids can be written as a product of two factors. One of the factors is $\delta_{\mathbf{K}_{\mathrm{D}}} \equiv \sigma_{0}(\boldsymbol{K})$ and this simply reflects the regularity of the void array. It is the same as the structure factor (3.1) for the array of vacancies. The other factor in $\sigma(\boldsymbol{K})$ for the void array, $F_{\mathrm{s}}(\boldsymbol{K})$, depends on the structure of the individual voids and not on their arrangement in space. It is just the structure factor of an isolated void, so that, in principle, $F_{\mathrm{s}}(\boldsymbol{K})$ contains information on the size and faceting of the voids.

The expression for the defect structure factor

$$
\begin{aligned}
\sigma(\boldsymbol{K}) & =\sigma_{0}(\boldsymbol{K}) F_{\mathrm{s}}(\boldsymbol{K}) \\
& =\delta_{\boldsymbol{K}, g_{\mathrm{D}}} F_{\mathrm{s}}(\boldsymbol{K})
\end{aligned}
$$

can be derived by noting that the sum over the empty lattice sites (2.5) can be written as a double sum over the $n_{\mathrm{v}}$ voids and the $\bar{n}$ vacant sites for each void. Thus the defect structure factor is

$$
\begin{aligned}
\sigma(\boldsymbol{K}) & =\frac{1}{n_{\mathrm{v}} \bar{n}} \sum_{\substack{\text { empty } \\
\text { sites }}} \exp \left(-\mathrm{i} \boldsymbol{K} \cdot \boldsymbol{R}_{m}\right) \\
& =\frac{1}{n_{\mathrm{v}}} \sum_{\substack{\text { voids } \\
i}} \frac{1}{\bar{n}} \sum_{\substack{\text { emply sites } \\
m \text { as void } i}} \exp \left(-\mathrm{i} \boldsymbol{K} \cdot\left(\boldsymbol{R}_{\boldsymbol{i}}+\boldsymbol{\rho}_{m}\right)\right]
\end{aligned}
$$

where $\boldsymbol{R}_{i}$ refers to the centre of the void, and $\rho_{m}$ is relative to this centre. 
Rewriting, the expression becomes

$$
\begin{aligned}
\sigma(\boldsymbol{K}) & =\left\{\frac{1}{n_{\mathrm{v}}} \sum_{\text {voids } i} \exp \left(-\mathrm{i} \boldsymbol{K} \cdot \boldsymbol{R}_{i}\right)\right\}\left\{\frac{1}{\bar{n}} \sum_{\substack{\text { empty sites } \\
\text { at one void }}} \exp \left(-\mathrm{i} \boldsymbol{K} \cdot \boldsymbol{\rho}_{m}\right)\right\} \\
& =\sigma_{0}(\boldsymbol{K}) F_{\mathbf{s}}(\boldsymbol{K})
\end{aligned}
$$

where $\sigma_{0}(\boldsymbol{K})$ is the same as in $(3.1)$ and $F_{s}(\boldsymbol{K})$ is

$$
\frac{1}{n} \sum_{\substack{\text { empty sites } \\ \text { at one void }}} \exp \left(-\mathrm{i} \boldsymbol{K} \cdot \boldsymbol{\rho}_{m}\right) \text {. }
$$

Equation (3.4) explains one of the striking results of Sass and Eyre, since it predicts that only a few extra peaks should be observed. For an extra peak to be observed, $\sigma(\boldsymbol{K})$ must be large compared with the general background. Clearly $\sigma_{0}$ must be finite, which means $\boldsymbol{K}$ must correspond to a $\boldsymbol{g}_{\mathrm{D}}$. But $F_{s}(\boldsymbol{K})$ must also be sufficiently large. Since $F_{\mathrm{s}}(\boldsymbol{K})$ decreases rapidly away from reciprocal lattice vectors $g_{H}$ of the host lattice, the way in which the observed peaks cluster around the perfect host peaks can be readily understood. This is shown quantitatively in figure 1.

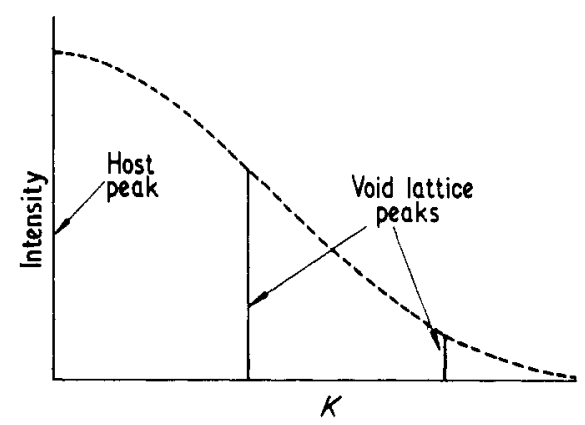

Figure 1. Intensities of void lattice spots. The predicted intensities are shown here for $K$ in the [110] direction and for a void spacing corresponding to the helium bubbles in Mo seen by Sass and Eyre. The dimensions of the helium bubbles are not accurately known. but the mean radius is of the order of 4 nearest neighbour distances, and is much less than that for the voids in neutron irradiated samples. In the figure a radius of 3.42 nearest neighbour distances was used.

\subsection{Dependence of $F_{s}(K)$ on void size and shape}

The void structure factor, $F_{s}(\boldsymbol{K})$, has been calculated numerically for a range of void sizes and shapes. One naturally exploits symmetry in performing the sum over sites, but no approximations are involved in the sum. Two limiting cases can be obtained analytically. For $\bar{n}=1$ one has isolated vacancies, and $F_{s}(K)=1$. For very large $\bar{n}$ if one can use a continuum limit in evaluating the sum, then

$$
F_{\mathrm{s}}(\boldsymbol{K})=3 \frac{\sin \chi-\chi \cos \chi}{\chi^{3}}
$$

for a sphere of radius $R=\chi /|\boldsymbol{K}|$. This second limit is important only for extrapolating some of the results for smaller voids. Comparing (2.4) and (3.4) one can see that $F_{\mathrm{s}}(\boldsymbol{K})$ tends to $S_{0}(K)$ at still larger $\bar{n}$. 
Values of $F_{\mathrm{s}}(\boldsymbol{K})$ have been obtained for voids of various sizes and for three types of shape: spherical, faceted along $\{110\}$, and faceted along $\{100\}$ (cubic). Some of the results for boc lattices are given in figures 1 to 3 .

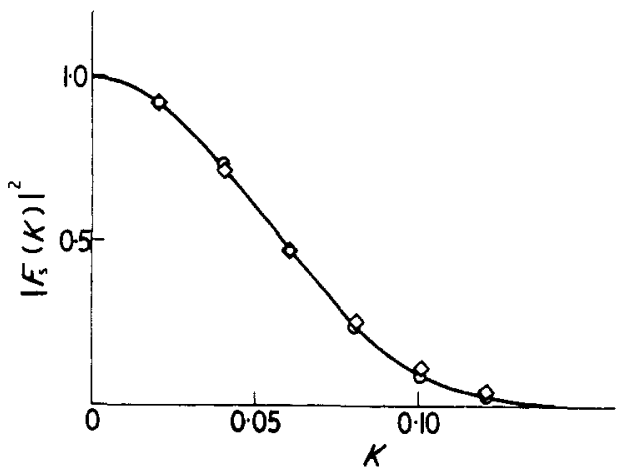

Figure 2. Effects of void shape on intensities. $\left|F_{s}(K)\right|^{2}$ is given for $K$ along the [110] direction, the units of $K$ are the separation of the adjacent spots for the perfect host lattice. The full line is for a spherical void of 363 vacancies. Points $\rightarrow$ are for a cubic void of 341 vacancies, and $O$ for a void with $\{110\}$ facets containing 369 vacancies. Similar results have been found for larger voids.

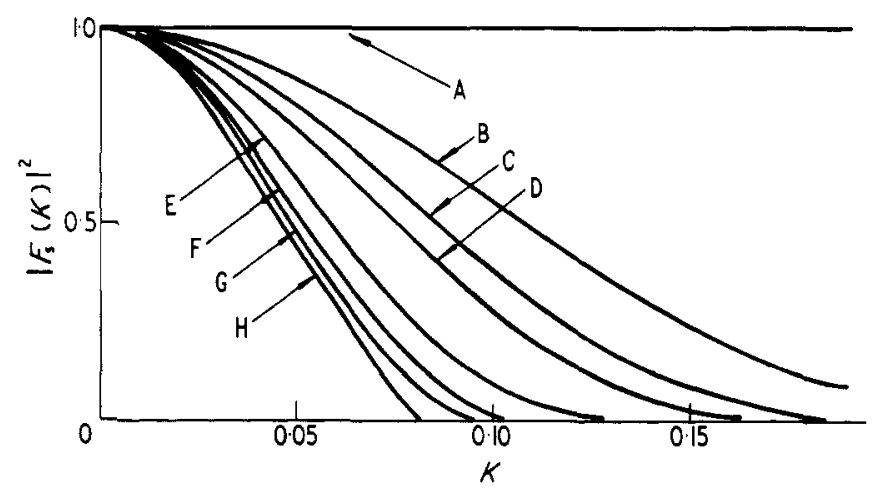

Figure 3. Variation of $\left|F_{\mathrm{g}}(K)\right|^{2}$ with void radius. The scattering vector $K$ is in the [110] direction, and given in units of the separation between adjacent spots for the perfect host lattice. The details of the voids $(\bar{n} \equiv$ the number of vacancies per void $(h . k . l) \equiv$ conrdinates of the outermost shell of vacancies, and $R \equiv$ the radius of the voids in units of the nearest separation of the host) are as follows :

\begin{tabular}{llrr}
\hline A & $\bar{n}=1$ & $(h . k, l)=(0,0,0)$ & $R=1 \cdot 0$ \\
B & 59 & $(2,2.2)$ & $2 \cdot 31$ \\
C & 113 & $(4,2,0)$ & $2 \cdot 83$ \\
D & 157 & $(4,4,0)$ & 3.42 \\
E & 363 & $(7,1,1)$ & $4 \cdot 16$ \\
F & 537 & $(8,0,0)$ & $4 \cdot 73$ \\
G & 645 & $(6,6,0)$ & $5 \cdot 00$ \\
H & 749 & $(8,4,0)$ & 5.26 \\
\hline
\end{tabular}


The first conclusion is that, for a given number of vacancies per void, the differences in $F_{s}(\boldsymbol{K})$ are far too small to provide a useful determination of void shape. There are slight differences from case to case, illustrated in figure 2 , but these will surely be masked by the limits of experimental resolution and crystal perfection.

The second conclusion is that the value $\boldsymbol{K}_{1 / 2}$ of the scattering vector for which $\left|F_{\mathrm{s}}(\boldsymbol{K})\right|^{2}$ is $\frac{1}{2}$ is roughly inversely proportional to the void radius. This is just the dependence expected from (3.5), for example. Quantitatively the results can be expressed by writing $\boldsymbol{K}_{\frac{1}{2}}$ in units of $\boldsymbol{K}_{\mathrm{H}}$, the difference between the adjacent host spot and its nearest [110] host spot. Writing the radius $R$ of a spherical void in terms of the nearest neighbour distance $a_{\mathrm{H}}$ for the host lattice. we find

$$
K_{1 / 2} R \simeq 0.24 K_{\mathrm{H}} a_{\mathrm{H}} \text {. }
$$

Figure 3 shows $\left|F_{s}(K)\right|^{2}$ for spherical voids of various sizes. It is worth noting that only very small scattering angles are involved, so that the variation of intensity with $K$ is determined almost entirely by the structure factor; the variation of the factor $V(\boldsymbol{K})$ is completely negligible. Thus equation (3.6) can be used to estimate the void size from observed intensity patterns. In principle, the void sizes can also be obtained by comparing the total intensities in the void lattice spots with those from the spots also present for the perfect hosts. Unfortunately, there are practical problems in measuring intensities, and neither method is likely to prove accurate quantitatively.

Whilst numerical values cannot be obtained easily, the intensities indicated in figure 1 are consistent with the experiment reported by Sass and Eyre. For their larger void spacing and for a void radius deduced from direct microscopy, a few very close peaks are expected. In practice, these are smeared together for other reasons, as observed by Sass and Eyre. For the small helium bubbles, one spot is clearly resolved experimentally. This is consistent with figure 1 , for the second void lattice spot should have a low intensity comparable with the background level.

\subsection{A void array finite in one dimension}

The void arrays observed, whilst strictly three dimensional, are much more restricted in extent in one direction than in the other two perpendicular directions. The array can be thought of as a small number, $n_{p}$ say, of parallel planes of ordered voids. It is difficult to decide how many planes are present from direct image experiments, so here the possibility of a determination of $n_{\mathrm{p}}$ from diffraction data is discussed.

As in (3.4), the defect structure factor $\sigma(K)$ can be obtained by simple manipulation. It is convenient to introduce new variables: $\boldsymbol{K}_{\|}$and $\boldsymbol{K}_{\perp}$ to describe the components of $\boldsymbol{K}$ parallel and perpendicular to the normal of the $n_{\mathrm{p}}$ void planes, $g_{\mathrm{D}_{\perp}}$ to give the reciprocal lattice vectors for one of the void planes, and $R_{\|}$and $R_{\perp}$ to describe respectively the separation and relative parallel displacement of voids in the adjacent planes. The result is :

$$
\begin{aligned}
\sigma(\boldsymbol{K})=\delta_{\boldsymbol{K}_{\perp}} \boldsymbol{g}_{\mathrm{D}_{\perp}} F_{\mathrm{s}}(\boldsymbol{K}) \sigma_{\mathrm{p}}\left(\boldsymbol{K}_{\perp}, \boldsymbol{K}_{\|}\right) \\
\begin{aligned}
\sigma_{\mathrm{p}}\left(\boldsymbol{K}_{\perp}, \boldsymbol{K}_{\|}\right) & =\frac{1}{n_{\mathrm{p}}} \sum_{\substack{\text { planes } \\
i=1}}^{n_{\mathrm{p}}} \exp \left[-\mathrm{i}\left(\boldsymbol{K}_{\perp}, \boldsymbol{R}_{\perp}+\boldsymbol{K}_{\|} \cdot \boldsymbol{R}_{||}\right) \hat{\lambda}\right] \\
& =\frac{1}{n_{\mathrm{p}}} \sum_{\substack{\text { pianes } \\
i=1}}^{n_{\mathrm{p}}} \exp (-\mathrm{i} \Delta \lambda)
\end{aligned}
\end{aligned}
$$


where $\Delta$ is $\left[\boldsymbol{K}_{\perp} \boldsymbol{R}_{\perp}+\boldsymbol{K}_{\|} \boldsymbol{R}_{\|}\right]$. Apart from an unimportant phase factor, values of $\sigma_{\mathrm{p}}$ for various $n_{\mathrm{p}}$ are

$$
\begin{array}{ll}
n_{\mathrm{p}}=1 & \sigma_{\mathrm{p}}=1 \\
n_{\mathrm{p}}=2 & \sigma_{\mathrm{p}}=\cos \left(\frac{1}{2} \Delta\right) \\
n_{\mathrm{p}}=3 & \sigma_{\mathrm{p}}=(1+2 \cos \Delta) / 3 \\
n_{\mathrm{p}}=4 & \sigma_{\mathrm{p}}=\cos \Delta \cos \frac{1}{2} \Delta \\
n_{\mathrm{p}}=\infty & \sigma_{\mathrm{p}}=\delta_{\Delta, 2 n \pi} .
\end{array}
$$

Clearly, if the dependence on $\left(\boldsymbol{K}_{\perp} \boldsymbol{R}_{\perp}+\boldsymbol{K}_{\|} \boldsymbol{R}_{\|}\right) \equiv \Delta$ can be discerned, there is some possibility of estimating $n_{\mathrm{p}}$ provided the number of planes is small. Even at $n_{\mathrm{p}}=4$ the predicted diffraction peaks are becoming rather too similar to those for the infinite array to be useful.

If $n_{\mathrm{p}}$ is very small, say 1 or 2 one might hope to observe extra spots. Unfortunately, the only unexpected features observed correspond to streaking in the [010] directions in figure 1 of Sass and Eyre. These extra features cannot be explained by the finite number of planes since, for $\boldsymbol{K}_{\|}=0$ (as here), the selection rules on $\boldsymbol{K}$ are independent of $n_{\mathrm{p}}$.

In principle, it should be possible to estimate $n_{\mathrm{p}}$ by comparing in detail intensities of spots which are equivalent when $n_{\mathrm{p}}$ becomes infinite. This has not been done experimentally, and would appear to be difficult technically.

\section{Disorder and the void lattice}

Electron microscopy shows clearly that the void and bubble lattices are imperfect. Here we discuss briefly the various forms of disorder on the diffraction patterns. This is important because it is by diffraction, rather than by direct microscopy, that the earlier signs of ordering are observed. Indeed, from direct microscopy of the bubble lattice above (eg Sass and Eyre figure $3(a)$ ) it is very hard to tell if ordering has occurred, whereas it is very clear in diffraction.

For simplicity we discuss separately several types of disorder and attempt to isolate their main features. In the real system, all types will be present simultaneously, giving a much more complicated situation than for the simple amorphous or liquid systems often treated. All types of disorder will cause some broadening of the individual diffraction peaks, although this may be hard to separate from the broadening already present due to dislocations and to point defects (cf Krivoglaz 1969).

The first form of disorder comes from the differing sizes and shapes of individual voids, even when they are centred accurately on a regular lattice. The distribution in sizes has been discussed by Evans (1972), who has given histograms of void sizes distributions after various irradiation conditions. There is no direct evidence for shape variations for voids in the size range of interest, but observations on larger voids make such variations probable. The main effects of this form of disorder are on $F_{s}(\boldsymbol{K})$. Since the regularity of the void centres has not been disturbed, the positions of the void spots will be unaltered, although there may be some smearing. But the relative intensities of the spots, determined by $F_{s}(\boldsymbol{K})$ will be modified. The value of $\boldsymbol{K}_{\frac{1}{2}}$ will now reflect some average void radius. Simple models, based on (3.5) and analytic fits to the size distributions given by Evans, suggest that the inferred value of the void radius will be reduced by the spread.

The second form of disorder involves gaps in the void lattice. Inspection of any of 
the photographs from direct microscopy shows there are patches - sometimes a dozen void spacings across - in which there are no voids, or else where the voids are very different in size. The patches show no ordering, but are apparently randomly distributed. Their main effect on the diffraction is to broaden the individual peaks. If, in addition to these large patches, there are also systematically missing voids, then more pronounced effects may be seen. For example, regions where every second void was missing could contribute to the streaking towards [010] seen in Sass and Eyre's figure (16).

The third form of disorder involves voids being displaced from their regular lattice sites. For simplicity we ignore variations in void sizes and shapes, and assume that the void lattice is complete. The effects of displacements of voids are most easily seen by comparing the void lattice with a geometrically similar array of holes in a continuum. The structure factor for the perfect array continuum case consists of a set of equal spikes at scattering vectors $\boldsymbol{K}$ equal to reciprocal lattice vectors $g_{\mathrm{D}}$ of the defect lattice. The effect of the underlying host lattice structure in a perfect void lattice is then to alter the relative intensities of these spikes, enhancing those for $g_{\mathrm{D}}$ close to the host lattice vectors $g_{\mathrm{H}}$. Suppose now there are displacements of the voids without any significant changes in the distortion of the intervening host lattice. Then it is likely that the individual spots will be broadened to include contributions from $k$ close to $g_{\mathrm{D}}$, but that there will be no major changes in the relative intensities of the different spots.

The broadening of the individual spots can be treated in the continuum model. As is well known in the theory of liquids, for example, the broadening can give a measure of the pair distribution function of the scatterers (here the voids, or the holes in the continuum). If there is a void centred on the origin, then the pair distribution function, $P(r)$, is the probability that there is a void centred at $r$. It is readily shown that the structure factor in the continuum model is related to the Fourier transform of $P(r)$ :

$$
\sigma(\boldsymbol{k})=\frac{1}{N} \int \mathrm{d}^{3} \boldsymbol{r} \exp (\mathrm{i} \boldsymbol{k} \cdot \boldsymbol{r}) P(\boldsymbol{r}) .
$$

Thus, if the broadening from disorder in position of the voids dominates, the widths of the spots give a measure of the order of the void lattice. However, because of the effects of the underlying host lattice structure on the spot intensities, it is not possible to invert the data satisfactorily to deduce $P(r)$. For the normal void lattice, such an inversion would be superfluous anyway, since the individual voids can be seen directly. But for the bubble lattice (figure 3 of Sass and Eyre), where the spacings are very small, some information about the ordering would be useful. From the one set of spots observed, and in the absence of other information about the character of the displacements of the voids from their regular sites, one can merely argue that the fractional spread in nearest neighbour void separations is likely to be comparable with the observed fractional spread in scattering wavevector. For the Sass-Eyre data, the spread in separations of the helium bubbles appears to be about $20 \%$ of the mean separation.

\section{Conclusion}

Sass and Eyre showed that the diffraction measurements were of value both in estimating the void spacings in a lattice and in recognising when ordering had occurred. The diffraction method is particularly useful for smaller voids and void spacings.

The present calculations have discussed three main points. First, it has been shown 
that the observed positions and intensities agree with the predictions, and that the clustering of the observed void-lattice spots around the host spots is to be expected. Secondly, the possibilities of obtaining information about void faceting, void radii and numbers of planes of voids were discussed. Unfortunately, the accurate measurement of intensity profiles is difficult in practice, for the large spacings lead to small Bragg angles and limited resolution, and reliable results seem to be ruled out. Finally, the qualitative effects of the various forms of disorder in the void lattice were discussed, and their various effects analysed.

\section{Acknowledgments}

I am indebted to Dr B L Eyre for a number of valuable discussions.

\section{References}

Evans J H 1971 Radiation Effects 1055

1972 AERE Rep. R-6733

Eyre B L and Bartlett A F 1973 J. Nucl. Mater. 47 143-54

Eyre B L and Evans J H 1971 Voids in Reactor Materials ed S F Pugh, D I R Norris and M H Lorretto(BNES) pp 323-31

Krivoglaz M 1969 Theory of X-ray and Thermal Neutron Scattering by Real Crystals (New York: Plenum)

Kulcinski, G. L., Brimhall J L and Kissinger H E 1972 Radiation-Induced Voids in Metals ed J W Corbett and L C Ianello USAEC Rep. CONF-710 $601 \mathrm{pp} 449-78$

Sass S L and Eyre B L AERE Rep. 1973 R-7358

Wiffen F W 1972 Radiation-Induced Voids in Metals ed J W Corbett and L C Ianello USAEC Rep. CONF710601 pp 386-96

Ziman J M 1964 Principles of the Theory of Solids (Cambridge: Cambridge University Press) 